\title{
Diffusion-influenced reactions in a hollow nano-reactor with a circular hole
}

\author{
Francesco Piazza, ${ }^{* a}$ and Sergey Traytak ${ }^{b, c}$
}

\author{
Received Xth XXXXXXXXXX 20XX, Accepted Xth XXXXXXXXX 20XX \\ First published on the web Xth $X X X X X X X X X X 200 X$ \\ DOI: 10.1039/b000000x
}

Hollow nanostructures are paid increasing attention in many nanotechnology-related communities in view of their numerous applications in chemistry and biotechnology, e.g. as smart nanoreactors or drug-delivery systems. In this paper we consider irreversible, diffusion-influenced reactions occurring within a hollow spherical cavity endowed with a circular hole on its surface. Importantly, our model is not limited to small sizes of the aperture. In our scheme, reactants can freely diffuse inside and outside the cavity through the hole, and react at a spherical boundary of given size encapsulated in the chamber and endowed with a given intrinsic rate constant. We work out the solution of the above problem, enabling one to compute the reaction rate constant to any desired accuracy. Remarkably, we show that, in the case of narrow holes, the rate constant is extremely well-approximated by a simple formula that can be derived on the basis of simple physical arguments and that can be readily employed to analyze experimental data.

\section{Introduction}

Chemical processes at the nano-scale are central to many complex phenomena in a wide array of modern nanotechnological applications. For example, it has long been known that hollow nanostructures provide some advantages in a number of applications (fillers, pigments, coatings, catalysts etc.) because of their lower density $1+5]$. Furthermore, physical and chemical features of hollow nanostructures can nowadays be fashioned in a controllable manner for a wide range of sizes, shapes, materials and structural properties of the shells, including thickness, porosity, and surface reactivity. As a consequence, increasing attention has been paid over the last decade to the elaboration of different engineering methods for manufacturing hollow nano-objects of various kinds.

Among many different nanostructures, hollow spheres and capsules have stimulated great interest because of their potential applications in controlled drug delivery systems $\mathrm{s}^{2617}$, artificial cells ${ }^{8}$, catalysis ${ }^{910}$, lithium batteries ${ }^{11}$ and as compartments for confined reactions 10,12 .

It is clear that many important physical and chemical processes such as diffusion transfer and chemical reactions might be considerably influenced by spatial restrictions $13-18$. Hol-

a Université d'Orléans, Château de la Source, 45100, Orléans, France, Centre de Biophysique Moléculaire, CNRS-UPR4301, Rue C. Sadron, 45071, Orléans, France. Fax: +33238 631517; Tel: +33238 255653; E-mail: Francesco.Piazza@cnrs-orleans.fr

${ }^{b}$ Le STUDIUM ${ }^{\circledR}, 3 D$ av. de la Recherche scientifique, 45071, Orléans, France.

${ }^{c}$ Semenov Institute of Chemical Physics RAS,4 Kosygina St., 117977 Moscow, Russia. low nanostructures find specific applications relative to their bulk counterparts mostly due to pronounced size-dependent effects emerging from the confined geometry of the reaction volumes. Nanochemical processes occurring in confined geometries usually take place within nano-scale reaction compartments (often referred to as nanoreactors), whose typical dimensions are greater than the relevant reactants sizes 19220. For example, typical nanoreactors for drug delivery consist of hollow spheres with reflecting walls and encapsulated prodrug particles that are needed for the local production of the appropriate drug. The spherical shells of such nanoreactors have one or several holes allowing small particles, reacting with prodrugs, to penetrate inside the nanoreactor by passive diffusion ${ }^{6}$. Other kinds of hollow spherical yolk-shell nanoparticles sinthetized as delivery vehicles or nanoreactors rely on hierarchical porous structures ${ }^{21}$ or are engineered as thermosensitive nano-catalysts 13 .

Typical dimensions of reactants and compartments ensure that reactions occurring in hollow nanostructures and mesoporous materials are mostly diffusion-influenced. This kind of reactions play an important role in chemistry and biology, and appropriate mathematical theories are well established for reactions occurring in for unbounded domains ${ }^{22123}$. However, despite their great potential importance in many different applications, there are very few studies devoted to diffusioninfluenced reactions occurring within hollow spheres.

To the best of our knowledge, this problem was first discussed by Adam and Delbrück ${ }^{24}$. Later Tachiya studied the kinetics of diffusion-controlled reactions between particles encapsulated within a micelle to describe luminescence quench- 
ing and excimer formation 25 . The theory of irreversible, diffusion-influenced quenching reactions of the type $A+B^{*} \stackrel{k}{\rightarrow}$ $A+B$ occurring at partially absorbing sinks within a spherical cavity and at the cavity surface were developed by Bug et al 26 . Three possible schemes for the location of partially absorbing surfaces within a spherical cavity (acceptors in the center, at the surface and at both locations) were considered.

In another study, the somewhat similar problem of the desorption of a lipid molecule from a lipid vesicle and its incorporation into another vesicle at high acceptor concentrations was reduced to solving the diffusion equation inside two concentric spheres 27 . To this end, perfectly absorbing boundary condition were imposed on the large sphere and appropriate matching boundary conditions were used on the surface of the small sphere. Analogous calculations were performed by Lü and Bülow, who solved the diffusion equation in different hollow geometries featuring either impermeable or permeable inner cores 28 .

Recently, more sophisticated in silico schemes based on complex sets of coupled reaction-diffusion boundary problems have been introduced with the aim of understanding the cellular behavior of toxic foreign compounds. Methods motivated by homogenization techniques have been applied to make such problem treatable, yielding good agreement with experiments 29 . Along similar lines, the theory of irreversible diffusion-controlled reactions has been applied to describe reactions between substrates and enzymes in a whole-cell model ${ }^{30}$. However, the Smoluchowski reaction rate constant was used in this study, which is questionable when one takes into account the confined geometry of the cell and crowding effects.

Overall, many studies that investigated reactions within confined geometries did not take into consideration the structure of the outer surface, often featuring one of more apertures (e.g. circular pores). Generally speaking, diffusive problems in geometries of this kind are known as narrow escape problems 31 . Recently, Sheu and Yang generalized the diffusive narrow escape problem to a gating escape model, describing the escape process of a Brownian particle out of a spherical cavity through a circular gate on the surface 32 . The angular size of the aperture was described by a time-dependent function $\theta_{0}(t)$, so that the gate behaves like an absorbing or reflecting patch in the open and closed states, respectively.

Remarkably, as it is done in Ref. 32 , absorbing boundary conditions are usually imposed on the gate/patch with the aim of modeling the diffusive escape of a particle from a confined volume through a hole on its surface. As a consequence, these theories cannot describe free diffusion of particles through the hole. In fact, this would necessitate that the model accommodate for both the diffusion from the inside to the exterior and in the opposite direction. Moreover, the mean first passage time approach is a powerful tool to study diffusion in compact domains but it is not appropriate for diffusion in a cavity connected with an outer, unbounded domain.

The problem of leakage of Brownian particles through a narrow pore studied in Ref. ${ }^{33}$ is much closer to the problem of free diffusion through a hole, as the flux density of the source on the boundary was taken into account. However, the flux of diffusing particles was given by a prescribed function and therefore it cannot describe free diffusion of particles through the hole ${ }^{33}$. Along the same lines, Berezhkovskii and Barzykin studied the kinetics of diffusive escape from a cavity through a narrow hole in the cavity wall and successive reentry by a formal kinetic scheme for reversible dissociation 34 .

The diffusion-influenced binding to a buried binding site connected to the surface by a channel studied in Ref. ${ }^{35}$ is the closest problem to the subject of our study that can be found in the literaure. Nevertheless, this problem was only solved for the case of a conical pit with the aid of a constant-flux approximation or for all geometries where diffusion occurs in interior regions that are so narrow that the problem can be approximately considered as one-dimensional.

A thorough analysis of the literature showed that up to now there are no studies devoted to the theory of diffusioninfluenced reactions occurring in hollow spheres connected through a circular hole to the unbounded outer space containing an excess of diffusing particles in the bulk. This is the problem that we solve in this paper.

The paper is organized as follows. In Section 2 we present a detailed formulation of the problem at issue. The solution of the problem is described in section 3, where we compute the reaction rate constant. In Section 4 we discuss our results and we show that our problem can be considered as equivalent to a much simpler one in the case of very small apertures. The main conclusions of the paper and possible extensions of the theory are given in Sec. 5. The appendix contains the details of the calculation and the explicit expressions of the matrix equations obtained by a dual series relations approach.

\section{The problem}

Let us consider particles $B$ with bulk density $\rho_{B}$ diffusing into a randomly distributed 3D system of hollow spheres with immobile reactants $A$ (sinks) encapsulated inside them. We assume the hollow spheres to be either fixed in space or mobile but much larger than the size of $B$ particles, so that they can be considered as immobile. For the sake of simplicity, we treat hollow spheres as infinitely thin identical spherical shells $\left(S_{R}\right)$ of radius $R$ comprising one spherical sink $\left(S_{a}\right)$ of radius $a(a \leq R)$ and reaction surface $\partial \Omega_{a}$ each, and featuring an axially symmetric hole $\partial \Omega_{0}$ corresponding to a spherical cap of aperture $\theta_{0}$ (see Fig. 1). To make calculations simpler, we consider every sink to be concentric with the envelope hollow 


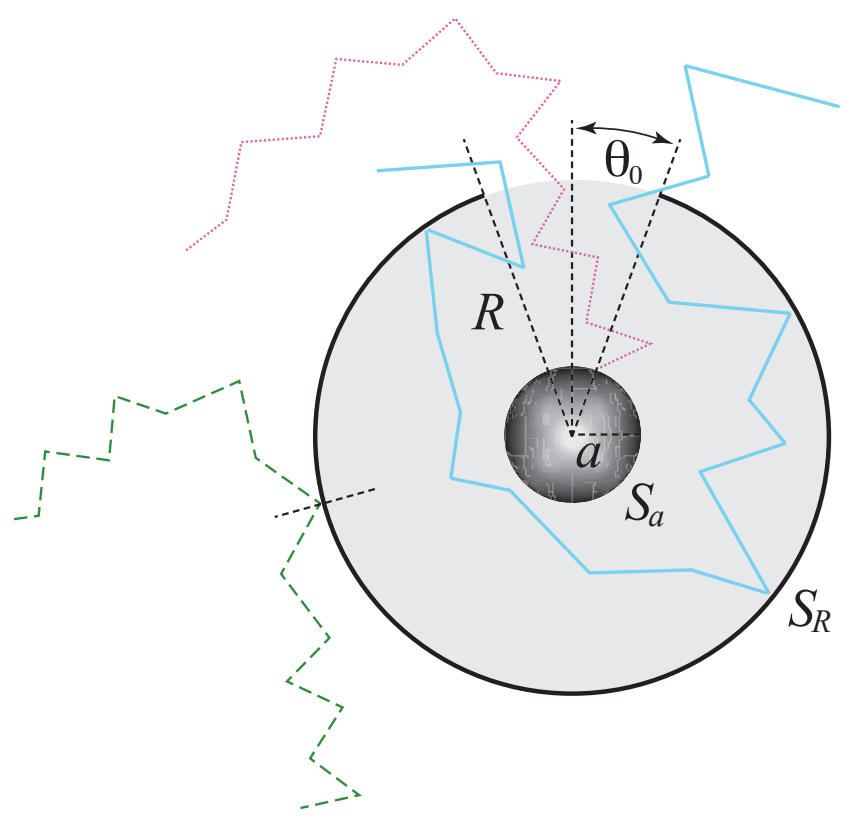

Fig. 1 (Color online) Schematic representation of our problem. Particles $B$ diffusing from the outside (diffusion coefficient $D_{\mathrm{o}}$ ) can be either reflected at the spherical surface $\mathscr{S}_{R}$ (dashed trajectory) or penetrate through the spherical cap hole. In the latter case, they diffuse with coefficient $D_{\mathrm{i}}$ and can be either absorbed at the inner spherical surface $\mathscr{S}_{a}$ (dotted trajectory) or diffuse back to the exterior through the hole (solid trajectory).

sphere ${ }^{*}$ Introducing a spherical coordinate system $(r, \theta, \varphi)$ with the origin at the center of the sphere, we have the following boundaries

$$
\begin{aligned}
& \partial \Omega_{0}=\left\{r=R, 0<\theta<\theta_{0}, 0 \leq \varphi<2 \pi\right\} \\
& \partial \Omega_{1}^{ \pm}=\left\{r=R \mp 0, \theta_{0}<\theta<\pi, 0 \leq \varphi<2 \pi\right\} \\
& \partial \Omega_{a}=\{r=a, 0<\theta<\pi, 0 \leq \varphi<2 \pi\}
\end{aligned}
$$

It is clear that, since the sphere is a two-sided surface, we deal with both the inside and outside boundaries: $\partial \Omega_{1}^{+}$and $\partial \Omega_{1}^{-}$ refer to limits taken from within the diffusion subdomains $\Omega^{+}$ and $\Omega^{-}$, respectively, where $\Omega^{+}=\{a<r<R, 0<\theta<\pi, 0 \leq$ $\varphi<2 \pi\}$ and $\Omega^{-}=\{R<r, 0<\theta<\pi, 0 \leq \varphi<2 \pi\}$.

In general, keeping in mind, e.g., biological applications, we should consider different media inside and outside the hollow sphere. Thus, we assume that the translational diffusion coefficient of particles $B$ can be approximated as

$$
D(\mathbf{r})=\left\{\begin{array}{llll}
D_{\mathrm{o}} & \text { in } & \Omega^{-} \cup \partial \Omega^{-} & \text {(outside) } \\
D_{\mathrm{i}} & \text { in } & \Omega^{+} \cup \partial \Omega^{+} & \text {(inside) }
\end{array}\right.
$$

\footnotetext{
* We note in passing that this constraint may be removed with the aid of reexpansion formulae methods 3637. In this way the solution of more general problems with sinks at arbitrary locations inside the hollow spheres is also feasible.
}

Let us also assume that the system relaxation time for the diffusive flux of $B$ particles $t_{D} \propto(R-a)^{2} / D_{\mathrm{i}}$ is small enough to neglect time-dependent effects. Hence, in the absence of external forces, the diffusion of particles $B$ with normalized number density $u(\mathbf{r})=\rho(\mathbf{r}) / \rho_{B}$ is described by the steadystate diffusion equation

$$
\nabla \cdot[D(\mathbf{r}) \nabla u(\mathbf{r})]=0 \quad \text { in } \Omega=\Omega^{+} \cup \Omega^{-} \cup \partial \Omega_{0}
$$

which should be solved with the customary bulk boundary condition

$$
\lim _{|\mathbf{r}| \rightarrow \infty} u(\mathbf{r})=1
$$

It is well known from the general theory of partial differential equations that the classical solution (twice continuously differentiable in $\Omega$ and continuous on $\bar{\Omega}$ ) of the stationary diffusion equation $(3)$ does not exist in the whole domain $\Omega$. Therefore one should consider the function

$$
u(\mathbf{r})=\left\{\begin{array}{lll}
u^{-}(\mathbf{r}) & \text { for } & \Omega^{-} \cup \partial \Omega^{-} \\
u^{+}(\mathbf{r}) & \text { for } & \Omega^{+} \cup \partial \Omega^{+}
\end{array}\right.
$$

Accordingly, continuity of the concentration field across the hole should be enforced, i.e.

$$
\left.u^{-}\right|_{\partial \Omega_{0}^{-}}-\left.u^{+}\right|_{\partial \Omega_{0}^{+}}=0
$$

Another condition on the hole can be derived by restricting to a small cylinder $\mathscr{C}_{\varepsilon}$ of section $\delta S \in \partial \Omega_{0}$ with its axis along the normal with height $\varepsilon \ll R$, i.e. $\mathscr{C}_{\varepsilon}=\{R-\varepsilon<r<$ $R+\varepsilon,(\theta, \varphi) \in \delta S\}$. Using Gauss-Ostrogradsky theorem and Eq. (3) one has

$$
\begin{aligned}
& \lim _{\varepsilon \rightarrow 0} \int_{\mathscr{C}_{\varepsilon}} \nabla \cdot[D(\mathbf{r}) \nabla u(\mathbf{r})] d^{3} \mathbf{r}= \\
& \quad=\int_{\delta S}\left[D_{\mathrm{i}} \frac{\partial u^{+}}{\partial r}-D_{\mathrm{o}} \frac{\partial u^{-}}{\partial r}\right] d S=0
\end{aligned}
$$

Since $\delta S$ is arbitrary, we obtain the following continuity condition for the local diffusion fluxes, holding at each point of the cap hole $\partial \Omega_{0}$,

$$
\left.\frac{\partial u^{-}}{\partial r}\right|_{\partial \Omega_{0}^{-}}=\left.\chi \frac{\partial u^{+}}{\partial r}\right|_{\partial \Omega_{0}^{+}}
$$

where we have introduced the diffusion anisotropy parameter

$$
\chi=\frac{D_{\mathrm{i}}}{D_{\mathrm{o}}}
$$

Conditions (6) and (8) for $D_{\mathrm{i}} \neq D_{\mathrm{o}}$ are often called the weak discontinuity conditions for the concentration field $u(\mathbf{r})$ † To

$\dagger$ Note that for $D_{\mathrm{i}}=D_{\mathrm{o}}$ the conditions (6) and 8 turn into continuity conditions for $u(\mathbf{r})$. 
complete the set of boundary conditions, the two-sided surface of the hollow sphere is assumed to be reflecting both from the inside $\partial \Omega_{1}^{+}$and from the outside $\partial \Omega_{1}^{-}$, i.e.

$$
\left.\frac{\partial u^{+}}{\partial r}\right|_{\partial \Omega_{1}^{+}}=\left.\frac{\partial u^{-}}{\partial r}\right|_{\partial \Omega_{1}^{-}}=0
$$

\subsection{The reaction rate constant}

We are interested in the pseudo-first-order irreversible bulk diffusion-influenced reaction between sinks $A$ (encapsulated in hollow spheres with a hole) and reactants $B$ freely diffusing in $3 \mathrm{D}$ space

$$
A+B \underset{k_{-D}}{\stackrel{k_{D}}{\rightleftharpoons}} A \cdot B \stackrel{k_{\text {in }}}{\longrightarrow} A+P
$$

where $A \cdot B$ denotes the so-called encounter complex, $k_{D}$ and $k_{-D}$ are the association and dissociation diffusive rate constants, respectively, and $k_{\text {in }}$ is the intrinsic rate constant of the chemical reaction occurring at the sink surface. Reactions of the kind (10) are customary dealt with by enforcing radiation boundary conditions ${ }^{\dagger}$ at the reaction surface $\partial \Omega_{a}$, i.e.

$$
\left[4 \pi a^{2} D_{\mathrm{i}} \frac{\partial u^{+}}{\partial r}-k_{i n} u^{+}\right]_{\partial \Omega_{a}}=0
$$

Thus, we can consider that hollow spheres effectively act as sinks of infinite capacity according to the pseudo-first-order reaction scheme

$$
A+B \stackrel{k_{a}}{\rightarrow} A+P
$$

where the forward diffusion-influenced rate constant $k_{a}$ is defined by the formula

$$
k_{a}=\left.\int_{\partial \Omega_{a}} D_{\mathrm{i}} \frac{\partial u^{+}}{\partial r}\right|_{r=a} d S
$$

Using this rate constant one can approximately describe the kinetics of the effective reaction (12) as

$$
c_{B}(t)=c_{B}(0) \exp \left(-k_{a} c_{A} t\right)
$$

where $c_{A}=$ const is the bulk concentration of hollow spheres, $c_{B}(t)$ is the time-dependent effective bulk concentration of $B$ particles. We stress that our schematization of the problem holds under the excess reactant condition $\rho_{A} \ll \rho_{B}$, $\rho_{A}$ being the bulk number density of sinks. Our goal is to compute the rate constant (13).

Equation (3) with the boundary conditions (4), (6), (8) and (11) completely specify our mathematical problem. It is expedient in the following to use the dimensionless spatial

\$ This kind of boundary conditions are also known as Robin boundary conditions. variable $\xi=r / R$. The problem at issue can be cast in the following form

$$
\begin{aligned}
& \nabla^{2} u^{ \pm}=0 \quad \text { in } \Omega^{ \pm} \\
& \left.\frac{\partial u^{+}}{\partial \xi}\right|_{\xi=\varepsilon}-h u^{+}(\varepsilon)=0 \quad \text { for } 0 \leq \theta<\pi \\
& \lim _{\xi \rightarrow \infty} u^{-}(\xi)=1 \\
& \left.\frac{\partial u^{ \pm}}{\partial \xi}\right|_{\xi=1^{\mp}}=0 \text { for } \theta_{0}<\theta<\pi \\
& \left.u^{+}\right|_{\xi=1^{-}}-\left.u^{-}\right|_{\xi=1^{+}}=0 \text { for } 0 \leq \theta \leq \theta_{0} \\
& \left.\chi \frac{\partial u^{+}}{\partial \xi}\right|_{\xi=1^{-}}-\left.\frac{\partial u^{-}}{\partial \xi}\right|_{\xi=1^{+}}=0 \quad \text { for } 0 \leq \theta \leq \theta_{0}
\end{aligned}
$$

where $\varepsilon=a / R$ and $h=k_{i n} R /\left(4 \pi a^{2} D_{\mathrm{i}}\right)$. The limit $h \rightarrow \infty$ corresponds to considering the boundary $\partial \Omega_{a}$ as a perfectly absorbing sink. In this case the reaction (10) becomes diffusionlimited, as the chemical conversion from the encounter complex $A \cdot B$ to the product $P$ becomes infinitely fast with respect to the diffusive step leading to the formation of $A \cdot B$.

\section{The solution}

We look for solutions in the form

$$
\begin{aligned}
& u^{-}(\xi)=1+\sum_{n=0}^{\infty} \frac{A_{n}}{\xi^{n+1}} P_{n}(\mu) \quad \text { for } \xi \geq 1 \\
& u^{+}(\xi)=\sum_{n=0}^{\infty}\left[\frac{B_{n}}{\xi^{n+1}}+C_{n} \xi^{n}\right] P_{n}(\mu) \text { for } \xi \leq 1
\end{aligned}
$$

where $A_{n}, B_{n}$ and $C_{n}$ are constants, $\mu=\cos \theta$ and $P_{n}(\mu)$ are Legendre polynomials of order $n$. Inserting eq. $16 \mathrm{~b}$ in eq. (15b), we get

$$
C_{n}=\alpha_{n} B_{n}
$$

with

$$
\alpha_{n}=\frac{n+1+h \varepsilon}{\varepsilon^{2 n+1}(n-h \varepsilon)}
$$

Formula (13) leads to the reduced reaction rate

$$
k_{a}^{*}=\frac{k_{a}}{k_{\mathrm{S}}^{+}}=\left.\frac{1}{2} \int_{-1}^{1} \frac{\partial u^{+}}{\partial \xi}\right|_{\xi=\varepsilon} d \mu
$$

where $k_{\mathrm{S}}^{+}=4 \pi D_{\mathrm{i}} a$ is the internal Smoluchowski rate constant for an ideal spherical sink of radius $a$. Inserting eq. (16b) in eq. (19) and making use of eqs. (17) and (18), we get

$$
k_{a}^{*}=-\frac{B_{0}}{\varepsilon}
$$

So the problem is reduced to the calculation of the constant $B_{0}$. The mixed boundary-value problem 1 (15a)-(15f) can be 
solved with the method of dual series relations (DSR) ${ }^{39}$. DSR admit solutions in the form of an infinite-dimensional system of algebraic equations for a new set of unknown coefficients $X_{n}, Y_{n}$, that are linearly related to $A_{n}, B_{n}{ }^{40}$

$$
Z_{n}=Z_{n}^{0}+\sum_{m=0}^{\infty} M_{n m} Z_{m}, \quad(n=\overline{0, \infty})
$$

Here $Z_{n}=\left(X_{n}, Y_{n}\right)^{T}, Z_{n}^{0}=\left(X_{n}^{0}, Y_{n}^{0}\right)$ and

$$
M_{n m}=\left(\begin{array}{cc}
M_{n m}^{11} & M_{n m}^{12} \\
M_{n m}^{21} & M_{n m}^{22}
\end{array}\right)
$$

where $M_{n m}^{i j}$ are four infinite-dimensional matrices of known elements, functions of the relevant geometrical and physical parameters $\varepsilon, h$ and $\chi$ (see appendix A for the details of the calculation and the explicit expressions of the matrices $M_{n m}^{i j}$ ). In particular, the expression for the rate as a function of the new coefficients is

$$
k_{a}^{*}=-\frac{Y_{0}}{\varepsilon}
$$

\section{Results and discussion}

Let us start by considering the limit $\varepsilon \rightarrow 1$, that is, $a \rightarrow R$. Furthermore, for the sake of simplicity, let us consider diffusionlimited reactions, i.e. $h \rightarrow \infty$. This case corresponds to considering a perfectly absorbing circular patch on an otherwise reflecting sphere of radius $R$. The rate constant for this system can be characterized by a steric factor $f_{R}\left(\theta_{0}\right) \in[0,1]$

$$
\frac{k_{R}}{k_{\mathrm{S}}^{-}}=f_{R}\left(\theta_{0}\right)
$$

where $k_{\mathrm{S}}^{-}=4 \pi D_{\mathrm{o}} R$ is the external Smoluchowski rate constant for an ideal spherical sink of radius $R$. The steric factor $f_{R}\left(\theta_{0}\right)$ can be calculated to any necessary accuracy with the DSR method ${ }^{40}$. In particular, it was found that

$$
f_{R}\left(\theta_{0}\right) \sim \frac{1}{2 \pi}\left(\theta_{0}+\sin \theta_{0}\right) \quad \text { as } \quad \theta_{0} \rightarrow 0
$$

In the general case $a<R$, it is expedient to normalize the reaction rate constant $k_{a}$ to the rate constant (24). This is tantamount to characterizing the sink inside the spherical cavity through a normalized effective steric factor $\hat{f}\left(\theta_{0} ; \varepsilon, \chi\right) \in[0,1]$, defined as

$$
\begin{aligned}
\hat{f}\left(\theta_{0} ; \varepsilon, \chi\right) & :=\frac{k_{a}}{k_{R}}=\frac{k_{a}}{k_{\mathrm{S}}^{-} f_{R}\left(\theta_{0}\right)} \\
& =\frac{\varepsilon \chi}{f_{R}\left(\theta_{0}\right)}\left(\frac{k_{a}}{k_{\mathrm{S}}^{+}}\right)
\end{aligned}
$$

The physical meaning of $\hat{f}\left(\theta_{0} ; \varepsilon, \chi\right)$ is to gauge how effective is the inner sink of radius $a$ in trapping a particle diffusing through the spherical cap hole with respect to the situation when the particle is instantaneously trapped the moment it touches the cap from the outside $(a=R)$. Indeed, as the sink grows to touch the internal wall of the cavity, one has

$$
\lim _{\varepsilon \rightarrow 1} \hat{f}\left(\theta_{0} ; \varepsilon, \chi\right)=1
$$

independently of $\chi$, as the inner sphere merges with the outer one. Conversely, as the sink shrinks, one has

$$
\lim _{\varepsilon \rightarrow 0} \hat{f}\left(\theta_{0} ; \varepsilon, \chi\right)=0
$$

uniformly with respect to $\chi$. In this case, the effective steric factor vanishes as there is no sink within the spherical cavity $\mathscr{S}_{R}$.

In Fig. 2 2 we plot the normalized effective steric factor as a function of the inner sink size $a$ for different values of the angular aperture of the circular hole. As the aperture decreases, $\hat{f}$ feels less and less the dependence on $a$, which appears to be limited to two boundary layers in the vicinity of $a=0$ and $a=R$. Between the two boundary layers, the effective steric factor is nearly constant.

In view of the Gauss-Ostrogradsky theorem, this is tantamount to saying that for small patches the inner sphere feels a constant flux on the surface $r=R$. Hence, the rate does not depend on the surface used for evaluating the integral 19 .

The value of $\hat{f}$ within the plateau is a measure of how much the whole system is less effective in trapping a tracer particle from the exterior with respect to the patched sphere $\mathscr{S}_{R}$. Therefore, it is a measure of the portion of incoming particle flux through the hole that does not reach the inner sink, e.g. the flux that escapes back to the exterior through the aperture in the cavity.

Interestingly, we see that the such value increases when the outside diffusion coefficient decreases with respect to the inside (increasing $\chi$ ). Recalling that the single-particle diffusion decreases in crowded environment due to the volume occupied by crowding agents, we conclude that, in order to reach diffusively the inner target more effectively through the hole, the inner medium should be less densely populated than the outside. This can be rationalized in terms of a reduced escape probability towards the exterior due to crowding. A different way to picture this effect is to recall that in the limit $\chi \rightarrow \infty$ the continuity condition (15f) turns the spherical hole into a perfectly reflecting patch from the interior. Again, no particles allowed to escape outside the spherical cavity.

The plateau value of the effective steric factor for small $\theta_{0}$ is proportional to the fraction of flux that reaches the inner sink at equilibrium, $\Phi_{\text {in }}$, while its complement to one is proportional to the flux $\Phi_{\text {out }}$ that leaves the inside of $\mathscr{S}_{R}$. As $D_{\mathrm{i}} / D_{\mathrm{o}}$ increases, we see that $\Phi_{\text {out }}$ goes to zero, meaning that 

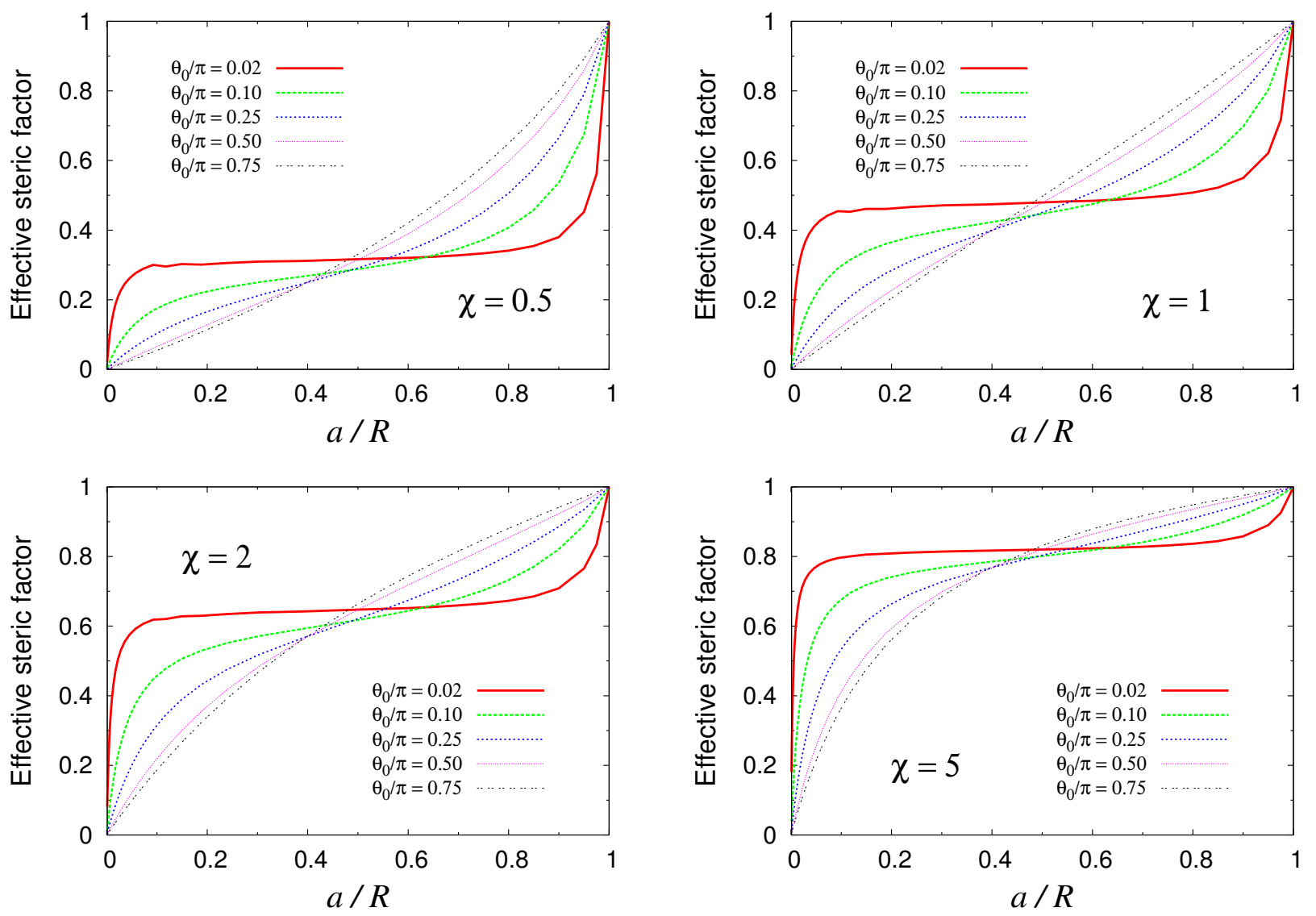

Fig. 2 (Color online) Plot of the normalized effective steric factor 26) as a function of the radius of the inner sphere for different sizes of the spherical cap hole and different values of $\chi$. The inner encapsulated sphere is taken as perfectly absorbing, i.e. the calculations are performed for $h \rightarrow \infty$.

particles become more and more trapped once they have diffused inside $\mathscr{S}_{R}$. A measure of $\Phi_{\text {out }}$ can thus be obtained by plotting the plateau value of $\hat{f}$ as a function of $\chi$. This is shown in Fig. 3 for the choice $a / R=0.5$ and $\theta_{0} / \pi=0.02$, so as to ensure that the boundary layers are sufficiently thin (see again bottom right panel in Fig. 2). We see that, for large values of the ratio $D_{\mathrm{i}} / D_{\mathrm{o}}$, the system behaves as a single sphere of radius $R$ with a small absorbing patch (practically no flux leaking back to the outside), i.e. $k_{a} \rightarrow k_{\mathrm{S}}^{-} f_{R}\left(\theta_{0}\right)$ (see again the definition (26).

The flux through the hole that reaches the sink is proportional to $D_{\mathrm{i}}$, while the incoming flux into the cavity is proportional to $D_{\mathrm{o}}$. We can thus surmise that, when the rate into the sink becomes independent of its size $a$ for small values of $\theta_{0}$, the fractions of flux reaching the sink and leaking back through the hole are approximately given by $D_{\mathrm{i}} /\left(D_{\mathrm{i}}+D_{\mathrm{o}}\right)$ and $D_{\mathrm{o}} /\left(D_{\mathrm{i}}+D_{\mathrm{o}}\right)$, respectively. This leads us to conjecture that the value of the effective steric factor (26) for sinks occupying the bulk region of the cavity, i.e. the plateau shown in Fig. 2 , is given by

$$
\hat{f}_{\text {plat }}=\frac{D_{\mathrm{i}}}{D_{\mathrm{i}}+D_{\mathrm{o}}}=\frac{\chi}{1+\chi}
$$

Fig. 3 shows that Eq. 29] yields a perfect interpolation of the plateau values, confirming the validity of our simple physical reasoning.

\subsection{Rationalizing the results through a simplified effec- tive model}

From the above discussion it should be clear that, for small $\theta_{0}$, we may model our system as a single sphere of radius $R$ endowed with a partially absorbing surface, characterized by an effective intrinsic reaction rate constant $k_{\text {eff }}$. This means that our boundary problem, for small values of the hole aperture, 


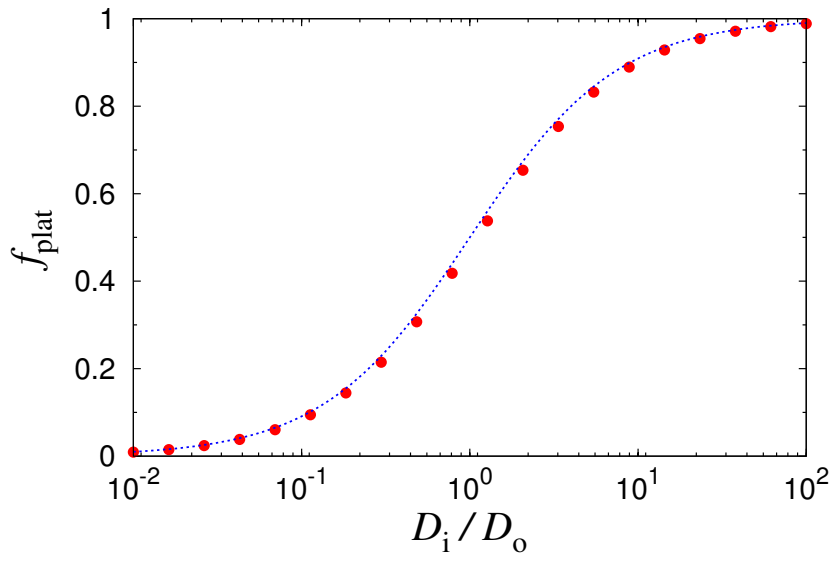

Fig. 3 (Color online) Plot of the plateau value of the normalized effective steric factor $26, \hat{f}_{\text {plat }}=\hat{f}\left(a / R=0.5, \theta_{0} / \pi=0.02\right)$ as a function of the inner-to-outer diffusivity ratio $\chi=D_{\mathrm{i}} / D_{\mathrm{o}}$ (symbols). The inner sphere is taken as a perfectly absorbing sink, i.e. the calculation is performed for $h \rightarrow \infty$. The dotted curve is a plot of the theoretical prediction, eq. 29].

should become equivalent to the following reduced problem

$$
\begin{aligned}
& \frac{d}{d \xi}\left(\xi^{2} \frac{d u}{d \xi}\right)=0 \\
& \left(\frac{\partial u}{\partial \xi}-h_{\mathrm{eff}} u\right)_{\xi=1}=0 \\
& \lim _{\xi \rightarrow \infty} u(\xi)=1
\end{aligned}
$$

The parameter $h_{\text {eff }}=k_{\text {eff }} / k_{\mathrm{s}}^{-}$gauges the effective absorbing power of the sphere $\mathscr{S}_{R}$. This should depend on the steric factor $f_{R}\left(\theta_{0}\right)$, which guarantees that only a portion of the surface is potentially absorbing by construction, and on $\chi=D_{\mathrm{i}} / D_{\mathrm{o}}$. It is easy to check that the rate constant $k$ for the above reduced problem is given by

$$
\frac{k}{k_{\mathrm{s}}^{-}}=\frac{h_{\mathrm{eff}}}{1+h_{\mathrm{eff}}}
$$

Recalling the definition (26), we see that eqs. (31) and 29) fix the effective reactivity of the reduced model, i.e.

$$
h_{\mathrm{eff}}=\frac{\chi f_{R}\left(\theta_{0}\right)}{1+\chi\left[1-f_{R}\left(\theta_{0}\right)\right]}
$$

\section{Conclusion and perspectives}

In this paper we investigated an irreversible, diffusioninfluenced reaction occurring within a spherical cavity endowed with a circular hole on its surface. Importantly, our model is not limited to small values of the angular aperture $\theta_{0}$ of the hole on the cavity surface. In our model, $B$ particles can freely diffuse inside and outside the cavity through the hole, and react at a spherical boundary $A$ encapsulated in the cavity and endowed with a given intrinsic rate constant. This model is relevant for chemical and biochemical reactions occurring in hollow nano-structures, which are intensively studied for a wide array of nanotechnological applications.

We work out the solution of the above problem, enabling one to compute the reaction rate constant for the encapsulated sphere within the cavity to any necessary accuracy.

Remarkably, we find that, for small values of the hole aperture, the rate constant $k_{a}$ becomes independent of the size of the inner reactive sphere. In this case, the rate is simply proportional to the fraction of diffusive flux that is actually absorbed by the sink and thus does not leak back through the hole into the bulk. We show how this situation can be encapsulated in a simple effective model, whose theoretical prediction provides a simple yet powerful formula, i.e.

$$
k_{a}=k_{\mathrm{S}}^{-} f_{R}\left(\theta_{0}\right) \frac{\chi}{1+\chi}
$$

Here $\chi=D_{\mathrm{i}} / D_{\mathrm{o}}$ is the ratio of the inside to outside diffusion coefficients, $k_{\mathrm{S}}^{-}=4 \pi D_{\mathrm{o}} R$ is the outside Smoluchowski rate constant into the spherical cavity and $f_{R}\left(\theta_{0}\right) \simeq\left(\theta_{0}+\right.$ $\left.\sin \theta_{0}\right) /(2 \pi)$ is the steric factor that characterizes the rate into the cavity when the hollow sphere is modeled as a perfectly absorbing patch (the hole) on an otherwise reflecting surface. Eq. 33) is a key result of this paper.

Future follow-ups of this work may include extending our mathematical framework to diffusion-influenced reactions with two axially symmetric hollow spheres and to situations where the encapsulated sink is no longer concentric with the hollow sphere but lies at an arbitrary location in the interior ${ }^{41}$.

\section{acknowledgement}

This research has been partially supported by Le STUDIUM $^{\circledR}$ (Loire Valley Institute for Advanced Studies), Grant No 2012-109. S. D. T. would like to thank P. Vigny and N. Fazzalari for their interest in this work. 
A

In this appendix we describe in detail the solution of the mixed boundary-value problem $15 \mathrm{a}$ - $-15 \mathrm{e}$ ) with the method of dual series relations (DSR).

The constants $A_{n}$ and $B_{n}$ can be determined by imposing the boundary conditions (15d) and the two continuity conditions (15e) and (15f). Recalling eq. (17), we get the two following coupled DSRs

$$
\begin{gathered}
\sum_{n=0}^{\infty}\left\{\left(1+\alpha_{n}\right) B_{n}-A_{n}-\delta_{n 0}\right\} P_{n}(\mu)=0, \\
0 \leq \theta \leq \theta_{0} \\
\sum_{n=0}^{\infty}(n+1) A_{n} P_{n}(\mu)=0, \quad \theta_{0}<\theta<\pi \\
\sum_{n=0}^{\infty}\left\{(n+1) A_{n}-\chi\left[n\left(1-\alpha_{n}\right)+1\right] B_{n}\right\} P_{n}(\mu)=0, \\
0 \leq \theta \leq \theta_{0} \\
\sum_{n=0}^{\infty}\left[n\left(1-\alpha_{n}\right)+1\right] B_{n} P_{n}(\mu)=0, \quad \theta_{0}<\theta<\pi
\end{gathered}
$$

where $\delta_{i j}$ is the Kronecker delta. The above DSRs can be cast in canonical form by defining

$$
\begin{aligned}
X_{n} & =\left(\frac{n+1}{2 n+1}\right) A_{n} \\
Y_{n} & =\left(\frac{n\left(1-\alpha_{n}\right)+1}{2 n+1}\right) B_{n}
\end{aligned}
$$

which gives

$$
\begin{array}{lc}
\sum_{n=0}^{\infty} X_{n} P_{n}(\mu)=G(\theta) & 0 \leq \theta \leq \theta_{0} \\
\sum_{n=0}^{\infty}(2 n+1) X_{n} P_{n}(\mu)=0 & \theta_{0}<\theta<\pi \\
\sum_{n=0}^{\infty} Y_{n} P_{n}(\mu)=F(\theta) & 0 \leq \theta \leq \theta_{0} \\
\sum_{n=0}^{\infty}(2 n+1) Y_{n} P_{n}(\mu)=0 & \theta_{0}<\theta<\pi
\end{array}
$$

with

$$
\begin{aligned}
& G(\theta)=\sum_{m=0}^{\infty}\left[\frac{X_{m}}{2(m+1)}+\beta_{m} Y_{m}\right] P_{m}(\cos \theta)-\frac{1}{2} \\
& F(\theta)=\sum_{m=0}^{\infty}\left[\frac{2 m+1}{\chi} X_{m}-2 m Y_{m}\right] P_{m}(\cos \theta)
\end{aligned}
$$

and

$$
\beta_{m}=\frac{\left(1+\alpha_{m}\right)(2 m+1)}{2\left[m\left(1-\alpha_{m}\right)+1\right]}
$$

The DSRs (36a), (36b), (36c), and (36d) admit a formal solution in the form of the infinite-dimensional system of algebraic equations $\frac{39}{39}$

$$
\begin{aligned}
& X_{n}=\frac{\sqrt{2}}{\pi} \int_{0}^{\theta_{0}} d u \cos \left[\left(n+\frac{1}{2}\right) u\right] \frac{d}{d u} \int_{0}^{u} \frac{G(\theta) \sin \theta d \theta}{\sqrt{\cos \theta-\cos u}} \\
& Y_{n}=\frac{\sqrt{2}}{\pi} \int_{0}^{\theta_{0}} d u \cos \left[\left(n+\frac{1}{2}\right) u\right] \frac{d}{d u} \int_{0}^{u} \frac{F(\theta) \sin \theta d \theta}{\sqrt{\cos \theta-\cos u}}
\end{aligned}
$$

The integrals appearing in eqs. $39 \mathrm{a}$ and $39 \mathrm{~b}$ can be computed explicitly ${ }^{42}$, by noting that $\$$

$$
\int_{0}^{u} \frac{P_{m}(\cos \theta) \sin \theta d \theta}{\sqrt{\cos \theta-\cos u}}=\frac{2 \sqrt{2}}{2 m+1} \sin \left[\left(m+\frac{1}{2}\right) u\right]
$$

which finally gives

$$
\begin{gathered}
X_{n}=\sum_{m=0}^{\infty}\left(M_{n m}^{11} X_{m}+M_{n m}^{12} Y_{m}\right)+X_{n}^{0} \\
Y_{n}=\sum_{m=0}^{\infty}\left(M_{n m}^{21} X_{m}+M_{n m}^{22} Y_{m}\right)+Y_{n}^{0}
\end{gathered}
$$

where

$$
\begin{aligned}
& M_{n m}^{11}=\frac{1}{2(m+1)} \Phi_{n m}, \quad M_{n m}^{12}=\frac{\left(1+\alpha_{m}\right)(2 m+1)}{2\left[m\left(1-\alpha_{m}\right)+1\right]} \Phi_{n m} \\
& M_{n m}^{21}=\frac{2 m+1}{\chi} \Phi_{n m}, \quad M_{n m}^{22}=-2 m \Phi_{n m} \\
& X_{n}^{0}=-\frac{\Phi_{n 0}}{2} \quad Y_{n}^{0}=0 \\
& \Phi_{n m}=\frac{1}{\pi}\left[\frac{\sin (m+n+1) \theta_{0}}{m+n+1}\right. \\
&\left.\quad+\frac{\sin (m-n) \theta_{0}}{m-n}\left(1-\delta_{m n}\right)+\theta_{0} \delta_{m n}\right]
\end{aligned}
$$

Note that $\Phi_{n m}=0$ for $\theta_{0}=0$, which gives $Y_{0}=0$. Hence the rate vanishes in this limit, as it should. The other interesting limit is $\theta_{0}=\pi$, when the larger external sphere no longer exists. In this case it is easy to see that $Y_{0}=\left(\alpha_{0}+1-\chi\right)^{-1}$. However, in the limit $\theta_{0}=\pi$, one has to consider $D_{\mathrm{i}}=D_{\mathrm{o}}=D$, as the separation between the two spatial domains $r<R$ and $r \geq R$ becomes immaterial. Hence, recalling eqs. (18) and 23 , we get

$$
\frac{k}{k_{\mathrm{S}}}=-\frac{1}{\alpha_{0} \varepsilon}=\frac{h \varepsilon}{1+h \varepsilon}=\frac{k_{\text {in }}}{k_{\mathrm{S}}+k_{\text {in }}}
$$

where $k_{\mathrm{S}}=4 \pi D a$, which is the correct result for a partially absorbing sphere with intrinsic reaction rate constant $k_{i n}$. The limit of fully absorbing sphere $k=k_{\mathrm{S}}$ is recovered in the limit $k_{\text {in }} \rightarrow \infty$.

$\S$ I. S. Gradshteyn and I. M. Ryzhik, Tables of Integrals, Series and Products, Academic Press, Eq. 7.225. 


\section{References}

1 Y. Yao, M. T. McDowell, I. Ryu, H. Wu, N. Liu, L. Hu, W. D. Nix and Y. Cui, Nano Letters, 2011, 11, 2949-2954.

2 Y. Chen, H. Chen, D. Zeng, Y. Tian, F. Chen, J. Feng and J. Shi, ACS Nano, 2010, 4, 6001-6013.

3 S. E. Skrabalak, J. Chen, Y. Sun, X. Lu, L. Au, C. M. Cobley and Y. Xia, Accounts of Chemical Research, 2008, 41, 1587-1595.

4 A. Srivastava, O. N. Srivastava, S. Talapatra, R. Vajtai and P. M. Ajayan, Nat Mater, 2004, 3, 610-614.

5 Y. Sun and Y. Xia, Analyst, 2003, 128, 686-691.

6 A. Ranquin, W. Versées, W. Meier, J. Steyaert and P. Van Gelder, Nano Letters, 2005, 5, 2220-2224.

7 S. Lehmann, S. Seiffert and W. Richtering, Journal of Colloid and Interface Science, 2014, 431, 204-208.

8 W. Li, X. Sha, W. Dong and Z. Wang, Chemical Communications, 2002, 2434-2435.

9 F.-P. Chang, Y.-P. Chen and C.-Y. Mou, Small, 2014, 10, 4785-4795.

10 B.-W. Park, D.-Y. Yoon and D.-S. Kim, Biosensors and Bioelectronics, 2010, 26, $1-10$.

11 M. Yang, J. Ma, C. Zhang, Z. Yang and Y. Lu, Angewandte Chemie, 2005, 117, 6885-6888.

12 J. Chattopadhyay, R. Srivastava and P. Srivastava, International Journal of Electrochemical Science, 2013, 8, 3740 - 3754.

13 S. Wu, J. Dzubiella, J. Kaiser, M. Drechsler, X. Guo, M. Ballauff and Y. Lu, Angewandte Chemie International Edition, 2012, 51, 2229-2233.

14 Y. Lu and M. Ballauff, Progress in Polymer Science, 2011, 36, 767-792.

15 A. Pich, W. Richtering, N. Welsch, M. Ballauff and Y. Lu, in Advances in Polymer Science, Springer Berlin Heidelberg, 2011, vol. 234, pp. 129163.

16 P. S. Burada, P. Hänggi, F. Marchesoni, G. Schmid and P. Talkner, ChemPhysChem, 2009, 10, 45-54.

17 O. Bénichou, C. Chevalier, J. Klafter, B. Meyer and R. Voituriez, Nature Chemistry, 2010, 2, 472-477.

18 Z. Konkoli, Proceedings Fifth Workshop on Developments in Computational Models \&\#8212; Computational Models From Nature, Rhodes, Greece, 11th July 2009, 2009, pp. 98-107.

19 H. Gröger, C. Kind, P. Leidinger, M. Roming and C. Feldmann, Materials,
2010, 3, 4355-4386.

20 H. J. Fan, U. Gösele and M. Zacharias, Small, 2007, 3, 1660-1671.

21 J. Liu, S. Z. Qiao, S. Budi Hartono and G. Q. M. Lu, Angewandte Chemie International Edition, 2010, 49, 4981-4985.

22 S. A. Rice, Diffusion-Limited Reactions, Elsevier, Amsterdam, 1985, vol. 25.

23 H.-X. Zhou, Quarterly Reviews of Biophysics, 2010, 43, 219-293.

24 G. Adam and M. Delbrück, Structural Chemistry and Molecular Biology, Freeman, S. Francisco, 1968.

25 M. Tachiya, Chemical Physics Letters, 1980, 69, 605 - 607.

26 A. L. R. Bug, E. L. Grossman, D. D. M. III and B. J. Berne, The Journal of Chemical Physics, 1992, 96, 8840-8852.

27 P. F. F. Almeida, Biophysical Journal, 1999, 76, 1922-1928.

28 Y. Lü and M. Bülow, Adsorption, 2000, 6, 125-136.

29 K. Dreij, Q. A. Chaudhry, B. Jernström, R. Morgenstern and M. Hanke, PLoS ONE, 2011, 6, e23128.

30 A. Vazquez, Journal of Theoretical Biology, 2010, 264, 356 - 359.

31 Z. Schuss, Journal of Scientific Computing, 2012, 53, 194-210.

32 S.-Y. Sheu and D.-Y. Yang, The Journal of Chemical Physics, 2000, 112, 408-415.

33 A. Singer, Z. Schuss and D. Holcman, Phys. Rev. E, 2008, 78, 051111.

34 A. Berezhkovskii and A. Barzykin, Chemical Physics Letters, 2004, 383, $6-10$.

35 A. M. Berezhkovskii, A. Szabo and H.-X. Zhou, The Journal of Chemical Physics, 2011, 135, 075103.

36 S. D. Traytak, Chemical Physics Letters, 1992, 197, 247 - 254.

37 S. D. Traytak and M. Tachiya, The Journal of Chemical Physics, 1997, 107, 9907-9920.

38 O. A. Ladyzhenskaya and N. N. Uralt'seva, Linear and Quasilinear Elliptic Equations, Academic Press, New York and London, 1968, vol. 46.

39 I. N. Sneddon, Mixed boundary value problems in potential theory, NorthHolland Pub. Co., 1966.

40 S. D. Traytak, Chemical Physics, 1995, 192, 1-7.

41 M.-V. Vazquez and L. Dagdug, Journal of Modern Physics, 2011, 2, 284 288.

42 F. Piazza, P. D. L. Rios, D. Fanelli, L. Bongini and U. Skoglund, European Biophysics Journal, 2005, 34, 899-911. 